\title{
Propagating waves in the sunspot umbra chromosphere
}

\author{
N. I. Kobanov and D. V. Makarchik \\ Institute of Solar-Terrestrial physics, Irkutsk, PO Box 4026, Russia \\ e-mail: kobanov@iszf.irk.ru \\ Received 31 December 2003 / Accepted 19 March 2004

\begin{abstract}
We investigate the line-of-sight velocity oscillations in the sunspot NOAA 0051 during its disk transit. The data obtained in this study provide evidence for the existence of running umbral waves in the chromosphere. These waves have a period of $2.8 \mathrm{~min}$ and propagate from the sunspot center outward with the phase velocity of $45-60 \mathrm{~km} \mathrm{~s}^{-1}$ with the line-of-sight velocity amplitude of about $2 \mathrm{~km} \mathrm{~s}^{-1}$. In most cases the waves terminate rather abruptly on the umbra boundary and show no direct linkage with running penumbral waves. The spatial coherence of the waves at the umbra center is no more than $2^{\prime \prime}$. At the photospheric level there are clearly pronounced periodic motions $(T \sim 5 \mathrm{~min})$ propagating from the inner penumbral boundary and from the superpenumbra to the lines of maximum Evershed velocity.
\end{abstract}

Key words. Sun: sunspots - Sun: photosphere - Sun: chromosphere - Sun: oscillations

\section{Introduction}

Despite the fact that the oscillations in sunspot umbra have been extensively studied over the last thirty years, the challenges involved have not declined in number (see Lites 1992; Bogdan 2000; Staude 1999). A most challenging problem involves ascertaining the origin of the three-minute oscillations in the chromosphere above the sunspot umbra and the connection of these oscillations with the wave processes in the penumbra (Balthasar 1987; Zhugzhda et al. 1985; Tsiropoula et al. 2000). Traditionally, the three-minute oscillations are thought of as being the manifestation of standing waves in the sunspot chromosphere. However, there is much discussion of identity of the three-minute oscillations with umbral flashes and as to whether these are responsible for the running penumbral waves (Tziotziou et al. 2002; Christopoulou et al. 2000, 2001). Upward propagating motions in the transition zone above the sunspot are discussed by Brynildsen et al. (2003). On the other hand, we still have little experimental data from which it would be possible to infer the character of the waves in the umbra chromosphere (standing or propagating) and measure directly their phase velocity in the former case.

\section{Observational data}

At the end of July - beginning of August 2002 we carried out a series of observations of the sunspot NOAA 0051. It was an isolated, near-round sunspot. The umbra-penumbra boundary was at $\sim 5^{\prime \prime}$ from the sunspot center, while the outer penumbra boundary was at $\sim 13^{\prime \prime}$. The observations were made with the horizontal solar telescope of the Sayan Observatory featuring the photoelectric guider that provides a tracking accuracy as high as one arcsec. The Princeton Instruments CCD-camera

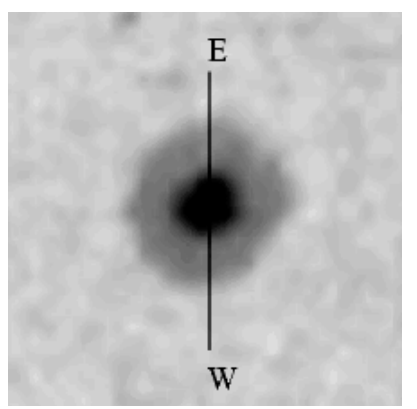

Fig. 1. Continuum image of NOAA 0051. The dark line shows the orientation of the spectrograph slit.

$(256 \times 1024)$, cooled to $-15^{\circ} \mathrm{C}$, was used. In the fifth spectral order, we recorded spectral regions with $\mathrm{H} \alpha$ and FeI $6569 \AA$ simultaneously. The image was rotated by means of the Dove prism in such a way that the spectrograph entrance slit intersected the sunspot center in the east-west direction (Fig. 1). In the spatial coordinate, $61^{\prime \prime}$ corresponded to 256 matrix pixels. The time interval between adjacent frames was $5 \mathrm{~s}$, and the duration of each series was $45 \mathrm{~min}$. A total of 10 time series was obtained for the period from July 27 to August 06 . For the $\mathrm{H} \alpha$ line, the line-of-sight velocity was calculated as the intensity difference in the red and violet wings, normalized to their sum, for three levels: $\pm 0.2 \AA ; \pm 0.4 \AA$, and $\pm 0.7 \AA$; for the FeI line, it was calculated only for one level, $\pm 0.05 \AA$. Instrumental spectral shifts were inferred from the telluric $\mathrm{H}_{2} \mathrm{O}$ line and then subtracted. The positive and negative velocity on all diagrams corresponds to the direction to and from the observer, respectively. On the half-tone diagrams the positive and negative velocities are represented by light and dark areas, respectively. 


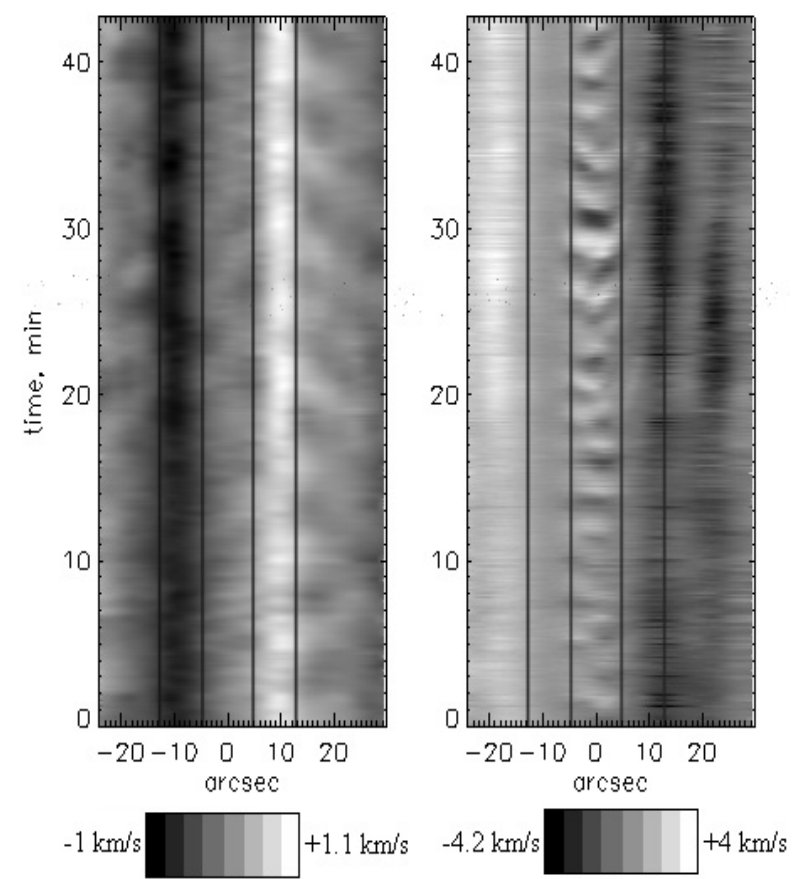

Fig. 2. Line-of-sight velocity as a function of position and time (NOAA 0051 time series - 09:08 UT on July 31, $\mu=0.95$ ), left panel photosphere (Fe I $6569 \pm 0.05 \AA$ ), right - chromosphere (H $\alpha \pm 0.2 \AA$ ). The vertical dimension from bottom to top is time (0-45 $\mathrm{min})$, the horizontal axis is distance from the spot center in arcsec. The vertical dark lines mark the penumbra boundaries.

\section{Results}

The first images of spatial-temporal distributions of the lineof-sight velocity in $\mathrm{H} \alpha$ showed a clear structure resembling a chevron. The position of the "chevron" along the time axis implies the presence of propagating wave motions in the sunspot umbra chromosphere (Fig. 2). These motions are directed from the umbra center outward.

It is difficult to imagine some artifact which would give rise to such a structure. Furthermore, in such a case the artifact influence would also be identical for the photosphere and the two distributions would appear identical, but they differ greatly from each other (Fig. 2). The "chevron" was so clear that it was possible to determine directly from it the period and the phase velocity $V=\Delta L / \Delta T$ of the propagating wave (Fig. 3). Upon averaging over the entire series, we obtained the values of period $2.8 \mathrm{~min}$ and $V=45-50 \mathrm{~km} \mathrm{~s}^{-1}$, respectively. The top of the "chevron" coincides with the umbra center in Fig. 2. For the points $\mathrm{B}$ and $\mathrm{C}$ in Fig. 3, the temporal distributions of the line-of-sight velocity in $\mathrm{H} \alpha \pm 0.2 \AA$ are plotted in Fig. 4. The line-of-sight velocity signal at the point $C$ is characterized by the mean time delay of $25 \mathrm{~s}$ with respect to the line-of-sight velocity signal at the point B. Since the distance between these points was taken to be $2^{\prime \prime}$, the phase velocity was slightly less than $60 \mathrm{~km} \mathrm{~s}^{-1}$, or a little more than the values inferred directly from the picture of the "chevron". It is interesting that Lites et al. (1982) measured the phase velocity as $60-70 \mathrm{~km} \mathrm{~s}^{-1}$ for the umbra and $20-35 \mathrm{~km} \mathrm{~s}^{-1}$ for the penumbra, but subsequently, Lites (1992) questioned the evidence. The maximum in the power spectrum corresponds to
C B

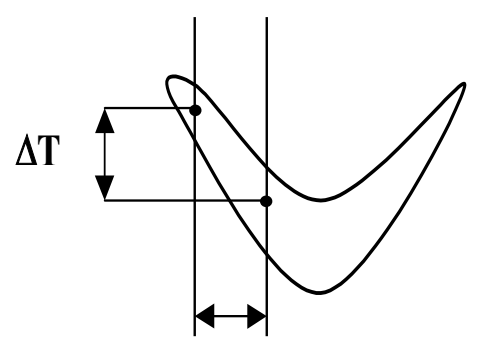

$\Delta \mathrm{L}$

Fig. 3. The schematic diagram for deducing of phase velocity.

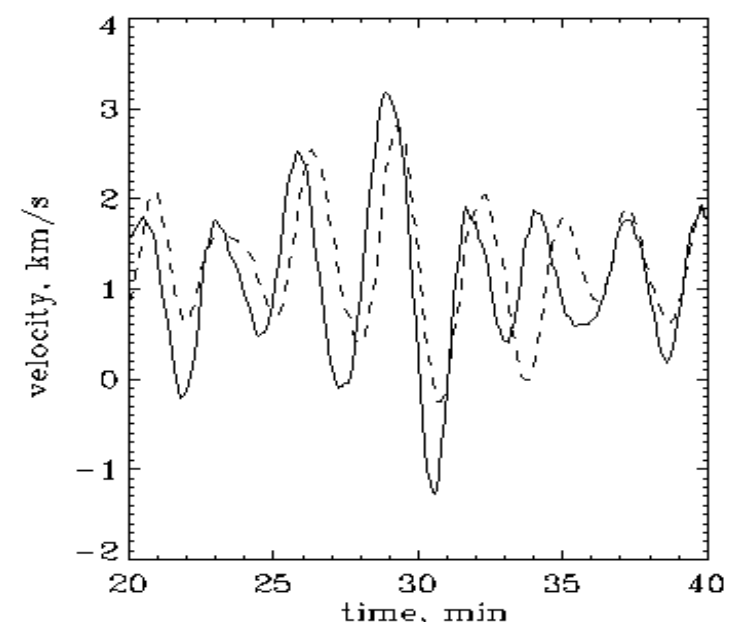

Fig. 4. The time delay between line-of-sight velocity signals $(\mathrm{H} \alpha \pm$ $0.2 \AA$ ) measured at the points C (dashed) and B (solid).

$5.7 \mathrm{mHz}$. The horizontal size of the region that is likely to be the source of wave motions and lies at the umbra center does not exceed 2 " according to preliminary estimates. No phase delay was found between line-of-sight velocity signals measured in $\mathrm{H} \alpha \pm 0.2 \AA$ and $\mathrm{H} \alpha \pm 0.7 \AA$ for a single spatial element. This would imply that the vertical extent of the source is comparable to the observed height scale (1000-1500 km); in any event it is no less than this value. Preliminary investigations showed that three-minute oscillations are very poorly pronounced in $\mathrm{H} \alpha$ intensity, and no "chevron" structure is present. In the first stage, no definite phase connection was revealed between the observed line-of-sight velocity and intensity oscillations.

Some authors (Bray 1973; Simon et al. 1982) insist that the center-of-gravity method for measuring velocity introduces errors (especially for the $\mathrm{H} \alpha$ line). Contrarily, Uitenbroek (2003) considers that this method is quiet reliable for measuring lineof-sight velocity and magnetic field strength. Note also that these errors mainly reduce to the fact that there is a zero shift of the velocity scale and that the absolute estimate of the velocity is distorted. This has no substantial influence on the establishment of the wave propagation, and on the determination of the phase velocity of the wave and the oscillation period.

A favorable concourse of circumstances (excellent seeing on July 31, 2002 plus intrinsic processes in the sunspot) allowed us to observe in some instances a clear picture of waves 

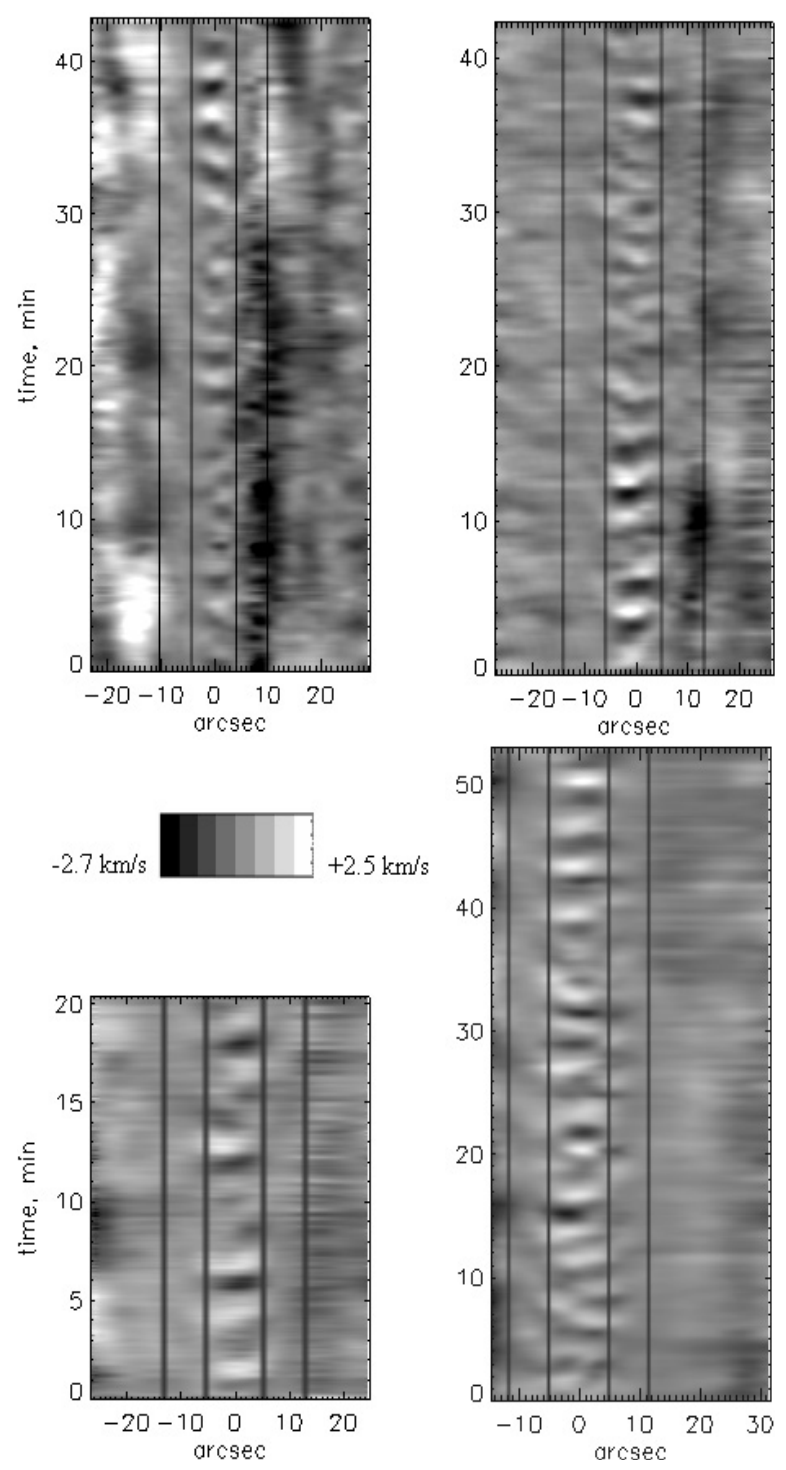

Fig. 5. Examples of the chevron structure in other time series for the same sunspot NOAA 0051. The chromospheric line-of-sight velocity $(\mathrm{H} \alpha \pm 0.2 \AA)$. The vertical dark lines mark the penumbra boundaries. Top: left - July 29, 05:22 UT ( $\mu=0.62)$; right - July 31, 03:30 UT ( $\mu=$ $0.89)$; bottom: left - August 1, 07:18 UT $(\mu=0.95)$; right - August 3, 09:08 UT $(\mu=0.93)$.

propagating in the umbra atmosphere. Thus it was possible to measure directly their phase velocity in the immediate region of sunspot umbra. Furthermore, the concurrent chromospheric processes were also accompanied by an interesting pattern of motions at the photospheric level. Unfortunately, the seeing was less favorable on the other days. Clear-cut chevron-shaped structures are present in some of the time series but not in all of them. Note also that the visibility of the chevron structure changes with the time even over the course of a single series of observations. While it is poorly pronounced in the initial time interval from 0 to $23 \mathrm{~min}$ (Fig. 2), it is most clearly seen between 23 and $43 \mathrm{~min}$. This can also be said about the other time series. Figure 5 presents portions of the observational series from July 29 and 31, 2002, and August 01 and 03, 2002, which validate this statement. Some of the portions in Fig. 5 show

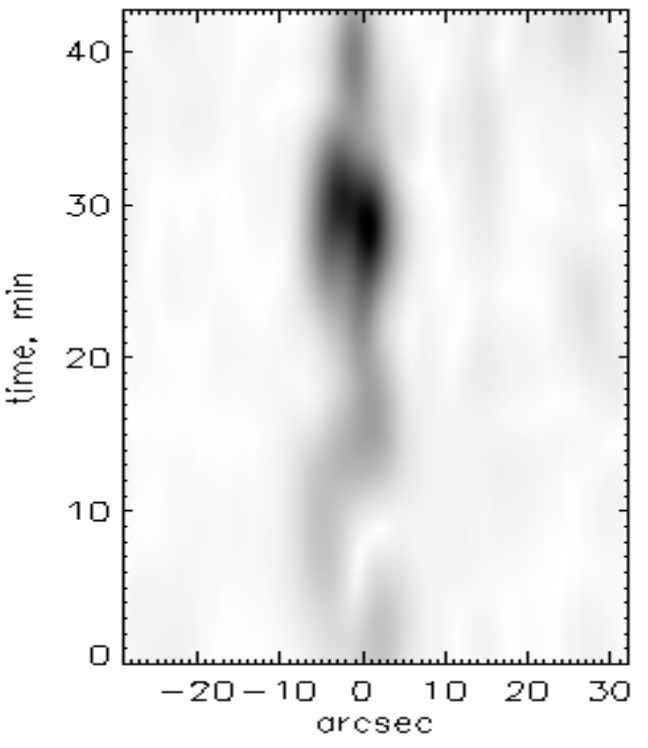

Fig. 6. Localization of chromospheric 3-min oscillations ( $\mathrm{H} \alpha \pm 0.2 \AA)$. "Space-time" wavelet diagram for the power of the $5.7 \mathrm{mHz}$ mode.

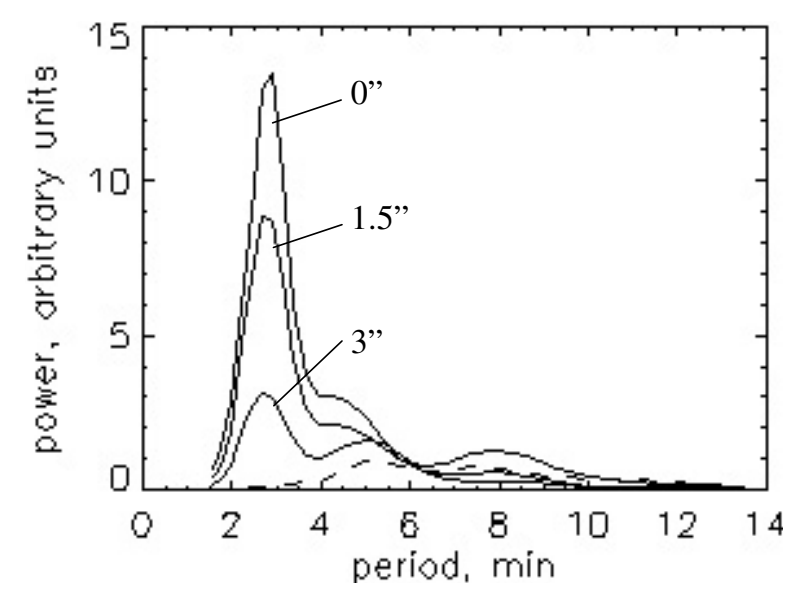

Fig. 7. The time-averaged power spectra of line-of-sight velocity oscillations ( $\mathrm{H} \alpha \pm 0.2 \AA$ ); the solid lines correspond to points inside the umbra $\left(0^{\prime \prime}, 1.5^{\prime \prime}, 3^{\prime \prime}\right.$ from center), the dashed line corresponds to the point near the inner boundary of the penumbra ( 7 " from center).

evidence of weak wave motion in the penumbra. The flattened "chevrons" in the sunspot umbra correspond to such structures. It may be suggested that this flattening is associated with an increase of the wave propagation velocity. A clear localization of the wave motions within the sunspot umbra boundaries that are inferred from the intensity of continuous spectrum is seen. The spatial size of the "chevron" $\left(11^{\prime \prime}\right)$ correspond to the umbra size $\left(10^{\prime \prime}\right)$. In observations from July 31 no indications were seen of the extension of the observed wave motions into the penumbra region - they terminate on the umbra boundary and immediately behind it; this is supported by both Figs. 2 and 6. It may be suggested that running penumbral waves are not discernible in Fig. 2 because of the small line-of-sight velocity amplitude $\left(1 \mathrm{~km} \mathrm{~s}^{-1}\right)$. But the direct and the inverse Evershed flows are clearly seen in Fig. 2, although their projections onto the line of sight give a close value $\left(1 \mathrm{~km} \mathrm{~s}^{-1}\right)$ because the sunspot lies nearly at the disk center. The power spectra in Fig. 7 show that 


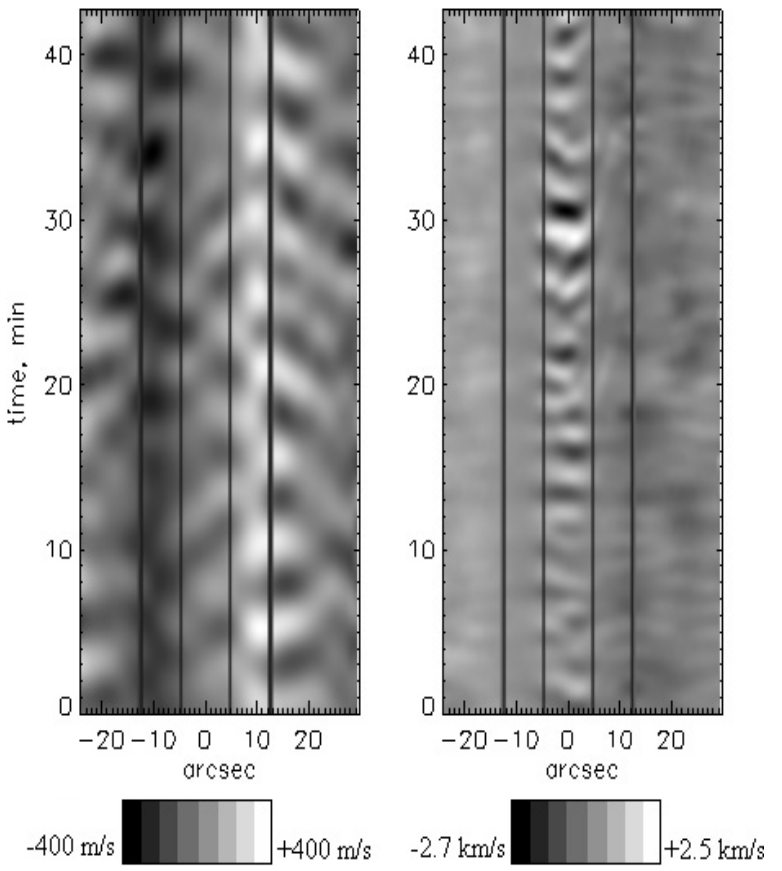

Fig. 8. The same as in Fig. 2, but the constant velocity components of the Evershed flows were decreased by a factor of 4 .

the oscillation power decreases with distance from the sunspot center. The 3-min period remains unchanged in the sunspot umbra, while inside the penumbra there appear indications of very weak oscillations (dashed line in Fig. 7) with a period of 5 min.

Figure 2 represents all motions in the sunspot umbra, both stationary (Evershed flows) and oscillatory. This is particularly handy for analyzing their possible linkage. However, to examine the weaker wave motions, it is necessary to decrease (or subtract) the constant velocity component of the Evershed flows. As a result, Fig. 2 is transformed to Fig. 8. As is evident from Fig. 8 (left panel), the pattern of motions at the photospheric level now is rather complicated even for a single sunspot of regular shape. In the first place, oscillatory motions in the sunspot umbra become visible; furthermore, one gets the impression that in some portions the Evershed flow itself is pulsating with a five-minute period. The wave structure and elements of Evershed flow in the photosphere extend beyond the outer penumbra boundary (Rimmele 1995; Kobanov 2000). Currently, it is difficult to interpret these data. Obviously, more extensive observational data are needed. The propagation of the umbral waves from the sunspot center in two opposite directions (Fig. 8, right panel) is not accompanied by the running penumbral waves in these same directions.

\section{Discussion and conclusions}

Is it true that the well-known umbral flashes (Beckers \& Tallant 1969; Moore \& Tang 1975) and our observed running umbral waves constitute the same phenomenon? There are some similar attributes: 1) the oscillation periods are close to $\sim 170 \mathrm{~s}$; the propagation velocities $\left(40 \mathrm{~km} \mathrm{~s}^{-1}\right.$ for umbral flashes, and 45-50 $\mathrm{km} \mathrm{s}^{-1}$ in the case under consideration) are also in reasonably good agreement; the size is no more than $3^{\prime \prime}$.
The phenomena are both observed within the sunspot umbra. The differences are: 1) in the case under consideration there are no brightenings in $\mathrm{H} \alpha$. Intensity oscillations are scarcely discernible and show no unambiguous connection with lineof-sight velocity oscillations. It is known, however, that some umbral flashes are not always accompanied by brightness variations in $\mathrm{H}$-alpha. Unfortunately, concurrent observations in CaII are not available to us (usually, they reveal intensity variations characteristic of umbral flashes). 2) For umbral flashes, the periods are different for different positions in the sunspot umbra, and several umbral flashes with different periods can be observed at a time. In the present case the period remains unchanged throughout the umbra observed. However, we only observed the slice in the East-West direction and so do not have the full picture.

Thus, we cannot discard the possible linkage of the observed waves with the known umbral flashes.

We next consider the possible connection of the observed phenomenon with running penumbral waves (Giovanelli 1972; Zirin \& Stein 1972). Giovanelli found that within sunspot umbra $(r=0.9)$ there are no radially propagating oscillations. Running waves, according to his observations, are produced within a narrow zone of the umbra (between 0.9 and $1 r$ ) and propagate outward with the velocity of $20 \mathrm{~km} \mathrm{~s}^{-1}$ in the sunspot penumbra. The period of these waves is about $300 \mathrm{~s}$, and the line-of-sight velocity amplitude is $1 \mathrm{~km} \mathrm{~s}^{-1}$.

Hence it is evident that our results differ. We observe radially propagating waves directly in the sunspot umbra. Moreover, these waves rather sharply terminate at the umbrapenumbra boundary or immediately nearby. The propagation velocity in the radial direction is $45-60 \mathrm{~km} \mathrm{~s}^{-1}$, the period is $\sim 170 \mathrm{~s}$, and the line-of-sight velocity amplitude averages $2 \mathrm{~km} \mathrm{~s}^{-1}$.

In later papers of a group of authors (Tsiropoula et al. 2000; Tziotziou et al. 2002) it is stated that running waves are generated within the umbra, and then propagate through the penumbra with a velocity of $20-30 \mathrm{~km} \mathrm{~s}^{-1}$. On the other hand, the authors do not address the problem of the difference of the periods (170 s in umbra, and $280-300 \mathrm{~s}$ in penumbra). The authors observed several oscillating elements in the sunspot umbra with their own periods and amplitudes. They even found some indication of oscillations with a period of $80 \mathrm{~s}$. They did not quantify the propagation velocity of the waves in the umbra. The authors believe that umbral oscillations and running waves have their origins in the same resonator.

Umbral flash-associated chromospheric oscillations were most thoroughly discussed by Rouppe et al. (2003). They suppose that the extreme brightness of the flash is caused by radiative transfer enhancement when the line of sight coincides with the propagation direction. Their results demonstrate that umbral flashes and running penumbral waves are closely related oscillatory phenomena.

Moore (1973) and Moore \& Tang (1975) claim that the sources of umbral oscillations and running penumbral waves are physically independent and lie in deeper subphotospheric layers. In Fig. 2 of our paper, we notice how drastically the spatial-temporal distribution of the line-of-sight velocity in the photosphere, measured in the line of FeI $6569 \AA$, differs from 
what we see in $\mathrm{H} \alpha$. In the umbra region there are hardly distinguishable oscillations or motions at the photospheric level (they become visible in Fig. 8). But on the other hand, the penumbra region shows a clear periodic structure whose center of symmetry is the line of maximum Evershed velocity. The period of this herringbone-like structure is about $5 \mathrm{~min}$; according to spectral analysis data, the refined period is $5.2 \mathrm{~min}$. From the inner part of the penumbra, and also from the outer penumbra the wave motions are directed opposite to each other. The waves appear as if they were flowing into the zone of maximum Evershed velocity directed to the observer. These results do not coincide with the new results of other group researchers (Georgakilas et al. 2000; Christopoulou et al. 2000, 2001). We do not rule out the possibility that the pattern of wave motions in the photosphere changes with time and is different for different sunspots.

Interestingly, Balthasar \& Wiehr (1990) developed the idea that the Evershed flows may be a result of superposition of some penumbral structure affected by the underlying oscillating layers. Their observational results for some sunspots show variety.

Thus, our "chevron" structure in the spatial-temporal distribution of the line-of-sight velocity, observed in the umbra chromosphere, strongly suggests the presence of propagating wave motions in the umbra chromosphere. These waves propagate from the sunspot center outward with the phase velocity of $45-60 \mathrm{~km} \mathrm{~s}^{-1}$ with a periodicity of $2.8 \mathrm{~min}$, and with the measured line-of-sight velocity amplitude of about $2 \mathrm{~km} \mathrm{~s}^{-1}$. In most cases the waves terminate rather abruptly at the inner boundary of the penumbra and do not propagate further into the penumbra. The spatial coherence of the motions does not exceed $2^{\prime \prime}$. Furthermore, the photospheric level shows periodic motions (with a period of $300 \mathrm{~s}$ ) directed from the inner boundary of the penumbra and from the superpenumbra to the line of maximum Evershed velocity.

We believe that the observed wave motions in the sunspot umbra chromosphere are not associated directly with running penumbral waves; but conceivably in some cases where their amplitude becomes sufficiently large $\left(6-8 \mathrm{~km} \mathrm{~s}^{-1}\right)$, which will be signaled by the occurrence of umbral flashes, oscillations with a period around $300 \mathrm{~s}$ will be driven on the umbrapenumbra boundary. The most intriguing fact is still the abrupt increase of the period and an equally abrupt decrease of the propagation velocity at the inner boundary of penumbra. It is hardly probable that this is a usual extension of the waves from umbra into penumbra. Most likely, on the inner boundary of the penumbra there is an abrupt transformation of the wave motions.

Another possibility could be associated with the penetration of our observed propagating waves with a five-minute period into the chromosphere from below (from the photosphere).

Numerical simulations of wave propagation in a stratified magneto-atmosphere presented by Bogdan et al. (2003) show that the coupling between the fast and slow magneto-acoustic-gravity waves is confined to thin atmospheric layers where the sound speed and the Alfen velocity are comparable in magnitude. Their coherent superposition presents a large variety of Doppler and intensity time series. Interestingly, some of their pictures (for example Fig. 31) resemble our "chevrons". In the future we will use information on the magnetic fields structure in observed sunspots.

Further observations (with longer time series) accompanied Ca II data and 2d-filtergramms in $\mathrm{H} \alpha$ are needed for better understanding of dynamic oscillations in sunspots.

Acknowledgements. This work was supported in part by the State Support of Leading Scientific Schools of the Russian Federation, grant SS-733.2003.2 and Federal "Astronomy" Program, grant No. 1105. We thank the anonymous referee for valuable remarks and suggestions that helped to improve the paper and Mr V. G. Mikhalkovsky for his help in preparing the English version of the manuscript.

\section{References}

Balthasar, H., Kuveler, G., \& Wiehr, E. 1987, Sol. Phys., 112, 37

Balthasar, H., \& Wiehr, E. 1990, A\&A, 237, 243

Beckers, J. M., \& Tallant, P. E. 1969, Sol. Phys., 7, 351

Bogdan, T. 2000, Sol. Phys., 192, 373

Bogdan, T., Carlsson, M., Hansteen, V., et al. 2003, ApJ, 599, 626

Bray, R. J. 1973, Sol. Phys., 30, 335

Brynildsen, N., Maltby, P., Kjeldseth-Moe, \& Wilhelm, K. 2003, A\&A, 398, L15

Christopoulou, E. B., Georgakilas, A. A., \& Koutchmy, S. 2000, A\&A, 354, 305

Christopoulou, E. B., Georgakilas, A. A., \& Koutchmy, S. 2001, A\&A, 375, 617

Georgakilas, A. A., Christopoulou, E. B., \& Koutchmy, S. 2000, A\&A, 363, 306

Giovanelli, R. G. 1972, Sol. Phys., 27, 71

Kobanov, N. I. 2000, Sol. Phys., 196, 129

Lites, B. W. 1992, in Sunspots: Theory and Observations, ed. J. H. Thomas, \& N. O. Weiss (Dordrecht: Kluwer), 261

Lites, B. W., White, O. R., \& Packman, D. 1982, ApJ, 253, 386

Moore, R. L. 1973, Sol. Phys., 23, 403

Moore, R. L., \& Tang, F. 1975, Sol. Phys., 41, 81

Rimmele, T. R. 1995, ApJ, 445, 511

Rouppe van der Voort, L. H. M., Rutten, P. J., Sutterlin, P., Sloover, P. J., \& Krijger, J. M. 2003, A\&A, 403, 277

Simon, G., Mein, P., Vial, J. C., Shine, R. A., \& Woodgate, B. E. 1982, A\&A, 115, 367

Staude, J. 1999, in Magnetic fields and oscillations, ed. B. Schmieder, A. Hofmann, \& J. Staude, ASP Conf. Ser., 184, 113

Tsiropoula, G., Alissandrakis, C. E., \& Mein, P. 2000, A\&A, 355, 375

Tziotziou, K., Tsiropoula, G., \& Mein, P. 2002, A\&A, 381, 279

Uitenbroek, H. 2003, ApJ, 592, 1225

Zhugzhda, Y. D., Locans, V., \& Staude, J. 1985, A\&A, 143, 201

Zirin, H., \& Stein, A. 1972, ApJ, 178, L85 